\title{
Clinical Practice and Guidelines for Managing Antithrombotics before and after Endoscopy: A National Survey Study
}

\author{
Seong Woo Jeon ${ }^{1}$, Su Jin Hong ${ }^{2}$, Soo Teik Lee ${ }^{3}$, Hyungkil Kim ${ }^{4}$, and Hoon Jai Chun ${ }^{5}$ \\ ${ }^{1}$ Department of Internal Medicine, School of Medicine, Kyungpook National University, Daegu, ${ }^{2}$ Department of Internal Medicine, \\ Soonchunhyang University College of Medicine, Bucheon, ${ }^{3}$ Department of Internal Medicine, Chonbuk National University Hospital, Jeonju, \\ ${ }^{4}$ Department of Internal Medicine, Inha University Hospital, Incheon, and ${ }^{5}$ Department of Internal Medicine, Institute of Gastrointestinal \\ Medical Instrument Research, Korea University College of Medicine, Seoul, Korea, Research Group for Endoscopic Submucosal Dissection in \\ Korean Society of Gastrointestinal Endoscopy
}

See editorial on page 275.

Background/Aims: The proper handling of antithrombotics is critical, and this study aimed to assess guideline adherence in the management of antithrombotics before and after endoscopy. Methods: A survey questionnaire was developed. The respondents' demographic information was included, and the questionnaire was divided into the first section for forceps biopsy, the second for polypectomy, and the third for endoscopic submucosal dissection (ESD) in which aspirin, clopidogrel, combination therapy (aspirin and clopidogrel), warfarin, and direct oral anticoagulants (apixaban) were prescribed to imaginary patients. Results: A total of 415 endoscopists completed this survey (response rate of $6.2 \%$, $415 / 6,673)$. The percentage of respondents who chose to proceed with biopsy for patients taking aspirin, those taking clopidogrel, those under combination therapy, those taking warfarin, and those taking apixaban was $89.4 \%$, $74.2 \%, 61.0 \%$, 38.6\%, and 50.4\%, respectively. Most respondents answered that they would discontinue aspirin, clopidogrel, and a combination of both drugs for 5 days before polypectomy or ESD (69.4\%/76.9\%, 83.6\%/83.9\%, and $53.3 \% / 65.8 \%$, respectively). The answers indicated that warfarin should be discontinued with heparin bridge therapy in high thromboembolic risk patients (polypectomy $70.1 \%$, ESD 73.5\%). Regarding apixaban use in polypectomy and ESD, $63.9 \%$ and $58.1 \%$ of respondents, respectively, chose answers consistent with the guidelines. Conclusions: The gap between the guidelines and clinical practice in the man- agement of antithrombotics before and after endoscopy is considerable and should be addressed via educational strategies. (Gut Liver 2020;14:316-322)

Key Words: Endoscopy; Guideline adherence; Antithrombotic agent

\section{INTRODUCTION}

Most developed countries are experiencing an increasingly aging society, and it is anticipated that there will be a concomitant rise in age-related comorbidities, including ischemic heart disease, cerebrovascular accidents, and malignancy. ${ }^{1}$ These patients are exposed to a greater risk of gastrointestinal bleeding and tend to be at elevated risk of bleeding during screening endoscopy or therapeutic endoscopy.

The cessation or continuation of antithrombotic agents is an important factor in ensuring safe endoscopy, and inappropriate decisions may have unintended consequences such as delayed bleeding or new thrombotic vascular events. ${ }^{2}$ There are a few national guidelines regarding the use of antithrombotic agents before and after endoscopy. ${ }^{3,4}$ Recently, a joint official statement by the Asian Pacific Association of Gastroenterology (APAGE) and the Asian Pacific Society for Digestive Endoscopy (APSDE) for the management of patients on antithrombotic agents who are undergoing emergency and elective endoscopy was published. ${ }^{5}$ These guidelines were developed in response to the increasing use of antithrombotics in patients undergoing gastrointestinal endoscopy in Asia. We reviewed these guidelines to determine the optimal timing of stopping or continu-

\footnotetext{
Correspondence to: Hoon Jai Chun

Department of Internal Medicine, Institute of Gastrointestinal Medical Instrument Research, Korea University College of Medicine, 73 Inchon-ro, Seongbuk-gu, Seoul 02841, Korea

Tel: +82-2-920-6555, Fax: +82-2-953-1943, E-mail: drchunhj@gmail.com

Received on April 23, 2019. Revised on May 17, 2019. Accepted on May 19, 2019. Published online November 11, 2019. pISSN 1976-2283 eISSN 2005-1212 https://doi.org/10.5009/gnl19133

(a) This is an Open Access article distributed under the terms of the Creative Commons Attribution Non-Commercial License (http://creativecommons.org/licenses/by-nc/4.0) which permits unrestricted non-commercial use, distribution, and reproduction in any medium, provided the original work is properly cited.
} 
ing antithrombotic agents before and after endoscopy. The current guidelines do not recommend stopping antithrombotic agents for low-risk procedures such as diagnostic endoscopy, with or without biopsies. However, the guidelines do recommend stopping these drugs for high-risk procedures, and these strategies are individualized by the type of drug and the risk of procedures. However, many complicating factors, such as fear of immediate or delayed bleeding, could hamper the consistent application of the guidelines to actual clinical practice. ${ }^{6}$

A few studies have assessed discrepancies between clinical practice and established guidelines. However, those studies were restricted to the management of aspirin, nonsteroidal antiinflammatory drugs, and low-risk endoscopic procedures. ${ }^{7-9}$ As of yet, no study based on multiple clinical situations ranging from forceps biopsy to endoscopic mucosal resection (EMR) or endoscopic submucosal dissection (ESD) has been published. We designed this survey according to various clinical scenarios after a thorough review of the APAGE and APSDE guidelines. The aim of this study was to assess adherence to the guidelines and evaluate the factors associated with differences between the guidelines and actual clinical practices.

\section{MATERIALS AND METHODS}

\section{Survey questionnaire}

A survey questionnaire was developed based on the APAGE and APSDE guidelines. ${ }^{5}$ The guidelines were thoroughly reviewed, and 18 questions pertaining to clinical scenarios with imaginary patients with various medical histories were formulated. The scenarios were reviewed and confirmed by a neurologist and cardiologist to achieve greater sophistication and better conformance to real clinical practice patterns. All of the authors were involved in the preparation of this questionnaire and confirmed the draft. The demographic information of the respondents, such as institution (clinic, hospital, general hospital, or tertiary hospital) and board certification (gastroenterology, surgery, family medicine, or others), was also assessed. The number of esophagogastroduodenoscopies (EGDs) or colonoscopies performed in their institution along with the number of EMRs or polypectomies and ESDs on a monthly basis was included. Respondents were asked to answer the questions based on their real clinical practice, not on their knowledge. The answer was chosen from the options given for each question; however, they were also allowed to directly write their answer in text form.

The pilot survey was first launched to the Korean ESD committee with 16 members. The members were asked to answer the questions, and they highlighted certain mistakes. Some changes were made to the draft, and a final version was prepared reflecting their opinions. The questionnaire was divided into three main parts. The first part comprised questions regarding the management of antithrombotic agents and the need for endoscopic forceps biopsy. There were five separate questions per- taining to the administration of aspirin, clopidogrel, combination therapy (aspirin and clopidogrel), warfarin, and direct oral anticoagulants (DOACs; apixaban). Similarly, the second part consisted of seven questions, in which imaginary patients in the need for colonoscopic polypectomy who had varying degrees of thromboembolic (TE) risk and had been taking drugs according to their clinical situations. The third part consisted of six different questions regarding the management of drugs before and after gastric ESD (Supplementary Table 1).

The final version was converted to an online document, and an invitation link was sent via e-mail. The e-mail addresses were extracted from the Korean Society of Gastrointestinal Endoscopy database, which has 6,673 members, and all of them were invited to answer the survey. Study data were collected by hypertext preprocessor tools managed by M2community (Seoul, Korea). A total of three e-mail invitation reminders were sent. The first e-mail was sent on July 27, 2018, and the last one on August 27, 2018. All data were collected anonymously.

\section{Statistics}

One of the authors (S.W.J.) managed the harvested data. Decisions described in the text were trimmed and allocated to one of the answer options provided where appropriate, for example, "stopping aspirin for 7 days" was merged with the given answer of "stopping aspirin for 5 days." Completely different opinions were counted as "other opinions." The proportion of answers consistent with the guideline was used as the main outcome variable. Predictors of answers consistent with the guideline such as the institution and the number of EGDs, EMRs, or ESDs were analyzed with a univariate analysis using the chi-square test. A p-value of $<0.05$ was considered significant.

\section{RESULTS}

\section{Baseline characteristics of the respondents}

A total of 415 endoscopists completed this survey (response rate, 6.2\%). Fifty-three percent of the respondents (222/415) were working in a general hospital or a tertiary hospital. With the exception of 15 respondents who were surgeons, family doctors, or general physicians, most of the respondents (96\%) were specialists in gastroenterology and gastrointestina endoscopy. In terms of institutional volumes, $41.7 \%$ of the respondents (173/415) belonged to the institutions performing more than 400 endoscopies or colonoscopies per month. EMR or polypectomy was performed more than 50 cases per month in $37.6 \%$ of the respondents. However, ESD was performed less than 10 cases per month in each hospital in $73.2 \%$ of the respondents (304/415), and was performed more than 50 cases in only $8 \%$ (33/415) (Table 1$)$.

\section{Scenario with forceps biopsy}

A clinical scenario of an active gastric ulcer around $10 \mathrm{~mm}$ in 
Table 1. Baseline Characteristics of the Respondents $(n=415)$

\begin{tabular}{|c|c|}
\hline Variable & No. (\%) \\
\hline \multicolumn{2}{|l|}{ Institution } \\
\hline Clinics & $121(29.2)$ \\
\hline Local hospitals & $72(17.3)$ \\
\hline General hospitals & $129(31.1)$ \\
\hline Tertiary hospitals & $93(22.4)$ \\
\hline \multicolumn{2}{|l|}{ Board certification } \\
\hline Gastroenterologist & $400(96.4)$ \\
\hline Surgeon & $2(0.5)$ \\
\hline General practitioner and others & $13(3.1)$ \\
\hline \multicolumn{2}{|c|}{ No. of EGD or colonoscopy per month } \\
\hline$<100$ & 73 (17.6) \\
\hline $100-200$ & 90 (21.7) \\
\hline $200-400$ & $79(19.0)$ \\
\hline$\geq 400$ & $173(41.7)$ \\
\hline \multicolumn{2}{|l|}{ No. of EMR per month } \\
\hline$<10$ & $93(22.4)$ \\
\hline $10-30$ & $122(29.4)$ \\
\hline $30-50$ & $44(10.6)$ \\
\hline$\geq 50$ & $156(37.6)$ \\
\hline \multicolumn{2}{|l|}{ No. of ESD per month } \\
\hline$<10$ & $304(73.2)$ \\
\hline $10-30$ & $51(12.3)$ \\
\hline $30-50$ & $27(6.5)$ \\
\hline$\geq 50$ & $33(8.0)$ \\
\hline
\end{tabular}

EGD, esophagogastroduodenoscopy; EMR, endoscopic mucosal resection; ESD, endoscopic submucosal dissection.

size was suggested. The choice to perform biopsy for a patient who had taken aspirin, clopidogrel, combination therapy (aspirin and clopidogrel), warfarin, or apixaban was indicated by $89.4 \%$, $74.2 \%, 61.0 \%, 38.6 \%$, and $50.4 \%$ of respondents, respectively. The positive response rate was the lowest in the scenario involving warfarin (Fig. 1).

\section{Scenario with simple colonic polypectomy}

A clinical scenario involving a $10-\mathrm{mm}$-sized pedunculated single colonic polyp in patients taking aspirin, clopidogrel, combination therapy, warfarin, or apixaban with varying degrees of TE risk was presented. Most respondents answered to discontinue aspirin, clopidogrel, or both drugs for 5 days before polypectomy (69.4\%, 83.6\%, and 53.3\%, respectively). The answer consistent with the guideline to continue aspirin or to continue aspirin but discontinue clopidogrel was selected by only $27.7 \%$ and $38.1 \%$ of respondents, respectively (Fig. 2). The answers consistent with the guideline indicating that warfarin should be discontinued for 5 days in patients with a low TE risk and for 5 days with heparin bridge therapy for patients with a high TE

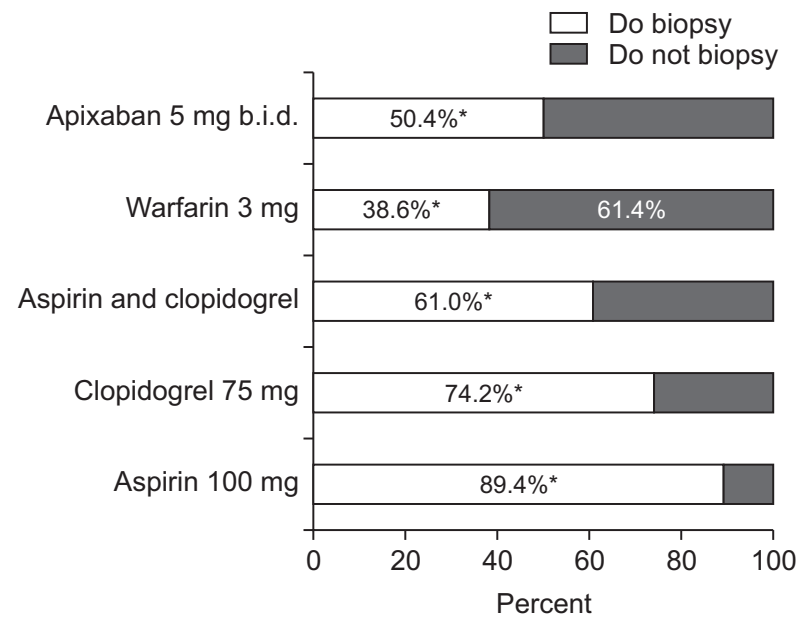

Fig. 1. The results of the survey section addressing the clinical scenario in which endoscopic forceps biopsy should be considered. The most frequently chosen answer was expressed as a percentage of all answers, and the asterisk $(*)$ indicates the recommendation in the guidelines.

b.i.d., twice a day.

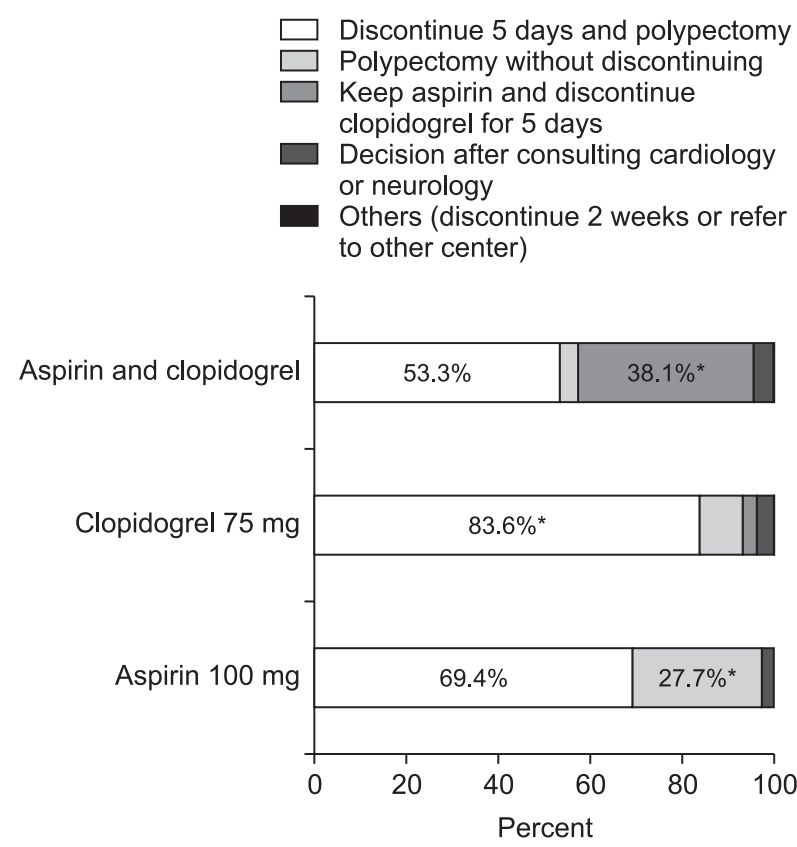

Fig. 2. The results of the survey section addressing the clinical scenario of polypectomy for patients taking aspirin, clopidogrel, or both drugs. The most frequently chosen answer was expressed as a percentage of all answers, and the asterisk $(*)$ indicates the recommendation in the guidelines.

risk were chosen by $47.0 \%$ and $70.1 \%$ of respondents, respectively. Regarding apixaban, 63.9\% of respondents selected the answer consistent with the guideline to discontinue the medication for 2 days. However, only 35.2\% of respondents chose the answer consistent with the guideline in a scenario of a high CHA2DS2-VASc score while taking warfarin (Fig. 3). 


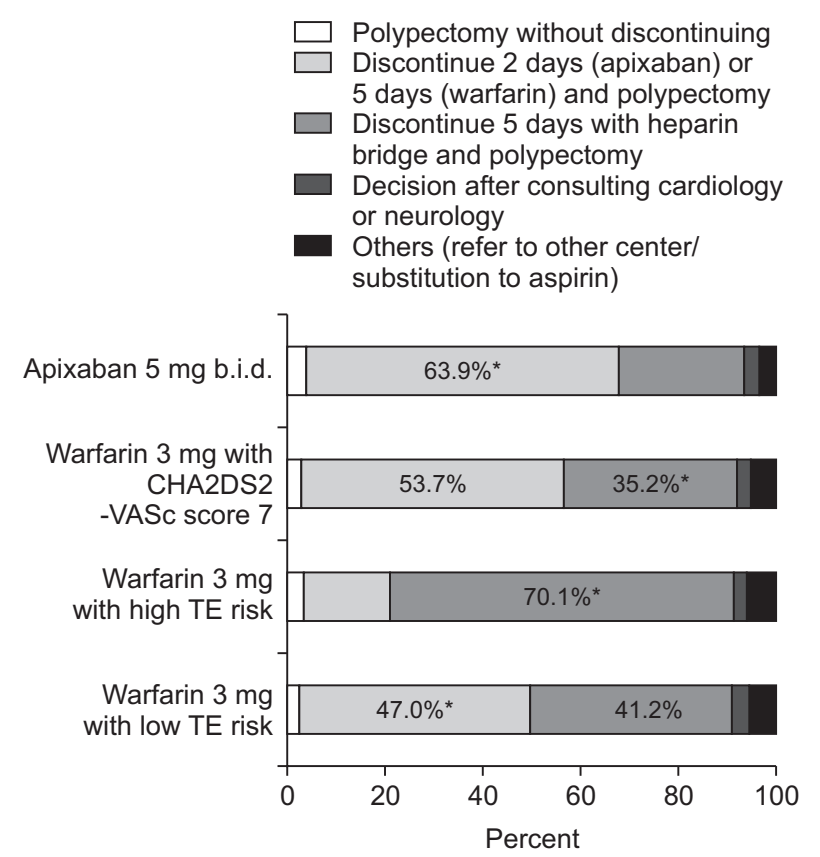

Fig. 3. The results of the survey section regarding the clinical scenario of polypectomy for patients taking warfarin and apixaban with varying degrees of thromboembolic (TE) risk. The most frequently chosen answer was expressed as a percentage of all answers, and the asterisk (*) indicates the recommendation in the guidelines. b.i.d., twice a day.

\section{Scenario with gastric ESD}

The final scenario involved gastric ESD cases with the same drugs and varying risk levels. The discontinuation of aspirin, clopidogrel, or both drugs for 5 days before ESD was favored by $76.9 \%, 83.9 \%$, and $65.8 \%$ of respondents, respectively. In a patient with high TE risk on warfarin, 73.5\% of respondents indicated that they would administer heparin bridge therapy; however, even in the context of low TE risk, 46.7\% of respondents preferred the use of heparin bridge therapy. More than half (58.1\%) of the respondents answered correctly with regard to the handling of apixaban in a gastric ESD patient (Fig. 4).

\section{Variables associated with the adherence to guidelines}

Variables such as the type of institution (clinic or local hospital vs general or tertiary hospital), number of EGDs or colonoscopies per month ( $<200$ vs $\geq 200$ ), number of EMRs or polypectomies per month ( $<30 \mathrm{vs} \geq 30$ ), and number of ESDs per month ( $<30$ vs $\geq 30$ ) were assessed to determine their effect on the answers chosen in each clinical scenario. Board certification was excluded from this analysis because most of the respondents were gastroenterologists. Most variables except the number of ESDs were associated with their intention to do biopsy in various situations, as well as colonic polypectomy and gastric ESD. The number of EMRs or polypectomies and ESDs was significantly associated with the correct response in each scenario
ESD without discontinuing

Discontinue 2 days (apixaban) or 5 days (aspirin, clopidogrel, warfarin) and ESD

$\square$ Keep aspirin and discontinue clopidogrel for 5 days

$\square$ Discontinue 2 days (apixaban) or 5 days (warfarin) with heparin bridge and ESD

$\square$ Decision after consulting cardiology or neurology

- Others (refer to other center/substitution to aspirin in warfarin or apixaban users)

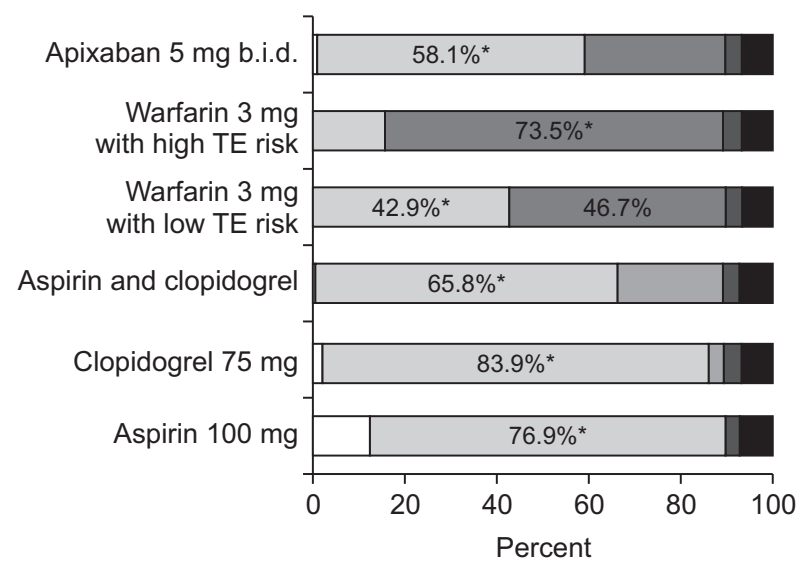

Fig. 4. The results of the survey section regarding the clinical scenario of gastric endoscopic submucosal dissection (ESD) for patients with varying degrees of thromboembolic (TE) risk. The most frequently chosen answer was expressed as a percentage of all answers, and the asterisk $(*)$ indicates the recommendation in the guidelines.

b.i.d., twice a day.

(Supplementary Tables 2-7).

\section{DISCUSSION}

Our study revealed some discrepancies between the established guidelines and actual clinical practice. The gap between the guidelines and clinical practice was lowest with regard to performing forceps biopsy for patients on aspirin and highest with regard to colonic polypectomy for patients on aspirin. The difference was also prominent in terms of polypectomy for patients on aspirin and clopidogrel, polypectomy for patients on warfarin with high CHA2DS2-VASc scores, and ESD for patients on warfarin with a low TE risk. There were no differences in the answers between experts and trainees in this survey; however, it revealed that factors such as the type of institution and volume of endoscopic procedures may affect decision-making in clinical practice.

The answers of more than half of the endoscopists demonstrated a willingness to perform biopsies in patients taking aspirin, clopidogrel, or a combination of the two drugs. However, half or less than half of the respondents indicated that they would skip biopsy procedures in patients taking DOAC or warfarin. The available guidelines define diagnostic EGD and 
colonoscopy with or without mucosal biopsy as low-risk procedures $^{3-5}$ for which there is no need to stop aspirin or clopidogrel. One prospective randomized study showed that no increased risk of hemorrhage from biopsy was found in patients receiving aspirin and clopidogrel. ${ }^{10}$ Moreover, the APAGE and APSDE guidelines do not recommend withholding warfarin if the INR is within the therapeutic range. ${ }^{5}$ DOACs are target-specific agents that are now frequently prescribed to patients and that have many advantages over vitamin $\mathrm{K}$ antagonists. ${ }^{11,12}$ Most guidelines, except for those in Europe,${ }^{4}$ indicate that there is no need to omit the morning dose of DOACs for forceps biopsy. However, less than half of the respondents were likely to do biopsy for warfarin users and only half of them for apixaban users. These results are interesting because the percentage of biopsies to be performed was higher in the context of apixaban administration than that of warfarin. This may be due to the fact that DOACs are more recently developed drugs, and many physicians may not be as familiar with the product name or pharmacodynamics of the drugs.

Simple polypectomy or colonic EMR is considered to carry a high risk of bleeding according to every guideline. ${ }^{3-5}$ However, available evidence shows that colonoscopic polypectomy has a low additional bleeding risk with aspirin and a high bleeding risk with clopidogrel. ${ }^{3}$ Indeed, a few studies have shown that the use of aspirin during polypectomy was not associated with an increase in bleeding. ${ }^{13,14}$ There was a high consistency rate for discontinuing clopidogrel but not aspirin in this study. The suggested clinical situation revealed a lower risk of TE even after stopping both drugs, which may have led to this result; however, the main reason was that most endoscopists are concerned about delayed bleeding after colonic polypectomy in patients taking aspirin. This result shows a typical feature of the gap between the guidelines and clinical practice. DOACs and warfarin showed considerable consistency with the guidelines, but many respondents answered incorrectly when asked about CHA2DS2-VASc scores. The CHA2DS2-VASc score system is useful in discriminating stroke risk in subjects with nonvalvular atrial fibrillation ${ }^{15}$ and is recommended to be used to estimate stroke risk in atrial fibrillation patients in the guidelines. ${ }^{16,17} \mathrm{In}$ our survey, more than half of the respondents indicated that they would stop warfarin without heparin bridge therapy, even in patients with high CHA2DS2-VASc scores of 7. This reveals that most endoscopists are not familiar with the use of this risk assessment scoring system in nonvalvular atrial fibrillation patients. The APAGE and APSDE guidelines recommend withholding DOACs for at least 48 hours before colonic polypectomy without bridging anticoagulation. However, 25.5\% of the respondents answered that they would perform bridging therapy in these patients, which may indicate a lack of awareness of the guidelines.

The response in the ESD scenario was highly consistent with the guidelines except for the answer to the question ad- dressing warfarin users with a low TE risk. A previous study suggested that delayed bleeding outcomes in ESD procedures were not impacted by whether or not antithrombotic agents were discontinued, ${ }^{18}$ while other studies revealed that the use of these agents was an independent risk factor for delayed bleeding. ${ }^{19,20}$ A recent meta-analysis also showed a higher frequency of delayed hemorrhages among patients taking antithrombotic agents. ${ }^{20}$ Although the European guidelines ${ }^{4}$ classify ESD as a high-risk procedure along with EMR, they recommend considering discontinuation of drugs such as aspirin in exceptional circumstances, depending on the individual risk factors. The Asian guidelines classify ESD as an ultrahigh-risk procedure and recommend discontinuation of antiplatelet agents. ${ }^{5}$ In terms of whether or not to discontinue antiplatelet drugs, most respondents chose the answer that corresponded to the guidelines. However, the question of whether warfarin should be taken or not revealed that the respondents failed to consider the TE risk, as they indicated that would do heparin bridge therapy even in patients with a low TE risk. For DOACs, 30.6\% of the respondents opted to administer heparin bridge therapy, demonstrating a lack of understanding of the drug and guidelines.

Although we were unable to ascertain the independent predictors of adherence to the guidelines, most of the variables, such as institution type and the number of EGDs, colonoscopies, EMRs, and ESDs, were associated with differences in the decision to continue or discontinue antithrombotics in each scenario. Therefore, the hospital volume and experience of the endoscopists clearly influence clinical decision-making. Following a guideline is never mandatory or binding and is not enforced, and practice guidelines are meant to foster a safer medical system. However, when guidelines are not followed, the integrity of the healthcare system may be at risk. ${ }^{21}$ In this study, there were conflicts between the guidelines and actual clinical practice. One study assessing discrepancies between clinical practice and guidelines with regard to antibiotics use before endoscopy suggested possible causes, including educational deficits, failure to publicize and/or justify updated practice guidelines, or views that current guidelines are flawed. ${ }^{22}$ In addition, another study on surveillance colonoscopy showed that reminding clinicians of certain guidelines is not sufficient to enhance adherence to guidelines. They concluded that identifying and eliminating all factors responsible for nonadherence to guidelines was necessary to achieve the goal of a safe, effective, and cost-effective colorectal cancer prevention tool. ${ }^{23}$ Further studies are necessary to evaluate the root causes underlying this problem and to initiate steps to improve compliance with current practice guidelines. Educational sessions addressing updates to practice guidelines, the implementation of policies consistent with practice guidelines, or the improved monitoring of adherence to guidelines $^{6}$ may enhance compliance. Finally, it is mandatory that unified guidelines be established based on solid evidence. ${ }^{24}$

This study has several limitations. First, the response rate 
was lower than expected, which may be due to the number and complexity of the questions. However, we were able to obtain enough information from this survey for each scenario. It is possible to assume that the low response rate might have underestimated the adherence to the guideline, since non-respondents might be less confident of their practice than respondents. Second, we were unable to determine the significant predictors of nonadherence to guidelines. However, this is a subject for further research entailing more detailed characteristics of the respondents, such as the extent of their training or educational status. Third, it is difficult to generalize these results to other nations because this survey was restricted to Korea. Further studies in other regions are warranted to elucidate discrepancies and enhance the adherence to guidelines.

In conclusion, there was a substantial gap between the guidelines and actual clinical practice in terms of the management of antithrombotics before and after endoscopy. Concerted efforts should be made to narrow the gap using targeted educational strategies.

\section{CONFLICTS OF INTEREST}

No potential conflict of interest relevant to this article was reported.

\section{ACKNOWLEDGEMENTS}

We thank all Endoscopic Submucosal Dissection Committee members of the Korean Society of Gastrointestinal Endoscopy and study personal for their contribution to the study.

\section{AUTHOR CONTRIBUTIONS}

Analysis and interpretation of data: S.W.J., H.J.C. Drafting of the manuscript, statistical analysis: S.W.J. Study design, administrative, technical and material support: H.J.C. Critical revision of the manuscript: H.J.C., S.J.H., S.T.L., H.K. Acquisition of data, study supervision: S.J.H., S.T.L., H.K. All authors read and approved the final manuscript.

\section{ORCID}

Seong Woo Jeon

$\mathrm{Su}$ Jin Hong

Soo Teik Lee

Hyungkil Kim

Hoon Jai Chun https://orcid.org/0000-0002-9539-9389 https://orcid.org/0000-0003-2012-0360 https://orcid.org/0000-0002-0758-1923 https://orcid.org/0000-0003-4230-3897 https://orcid.org/0000-0002-5539-361X

\section{REFERENCES}

1. Abraham NS. Gastrointestinal bleeding in cardiac patients: epidemiology and evolving clinical paradigms. Curr Opin Gastroenterol
2014;30:609-614.

2. Greenwald DA. Managing antithrombotic agents during endoscopy. Best Pract Res Clin Gastroenterol 2016;30:679-687.

3. ASGE Standards of Practice Committee, Acosta RD, Abraham NS, et al. The management of antithrombotic agents for patients undergoing GI endoscopy. Gastrointest Endosc 2016;83:3-16.

4. Veitch AM, Vanbiervliet G, Gershlick AH, et al. Endoscopy in patients on antiplatelet or anticoagulant therapy, including direct oral anticoagulants: British Society of Gastroenterology (BSG) and European Society of Gastrointestinal Endoscopy (ESGE) guidelines. Gut 2016;65:374-389.

5. Chan FKL, Goh KL, Reddy N, et al. Management of patients on antithrombotic agents undergoing emergency and elective endoscopy: joint Asian Pacific Association of Gastroenterology (APAGE) and Asian Pacific Society for Digestive Endoscopy (APSDE) practice guidelines. Gut 2018;67:405-417.

6. Schoenfeld P. Why don't endoscopists follow guideline recommendations and how can we improve adherence? Endoscopy 2018;50:93-95.

7. Lee SY, Tang SJ, Rockey DC, et al. Managing anticoagulation and antiplatelet medications in GI endoscopy: a survey comparing the East and the West. Gastrointest Endosc 2008;67:1076-1081.

8. Ono S, Fujishiro M, Kodashima S, et al. Controversy on the management of anticoagulants and antiplatelet agents for scheduled endoscopy. Dig Endosc 2011;23:1-4.

9. Feuerstein JD, Tapper EB, Belkin E, et al. Multicenter study assessing physician recommendations regarding the continuation of aspirin and/or NSAIDs prior to gastrointestinal endoscopy. Dig Dis Sci 2015;60:3234-3241.

10. Whitson MJ, Dikman AE, von Althann C, et al. Is gastroduodenal biopsy safe in patients receiving aspirin and clopidogrel? A prospective, randomized study involving 630 biopsies. J Clin Gastroenterol 2011;45:228-233

11. Granger CB, Alexander JH, McMurray JJ, et al. Apixaban versus warfarin in patients with atrial fibrillation. N Engl J Med 2011;365:981-992.

12. Patel MR, Mahaffey KW, Garg J, et al. Rivaroxaban versus warfarin in nonvalvular atrial fibrillation. N Engl J Med 2011;365:883891.

13. Hui AJ, Wong RM, Ching JY, Hung LC, Chung SC, Sung JJ. Risk of colonoscopic polypectomy bleeding with anticoagulants and antiplatelet agents: analysis of 1657 cases. Gastrointest Endosc 2004;59:44-48.

14. Manocha D, Singh M, Mehta N, Murthy UK. Bleeding risk after invasive procedures in aspirin/NSAID users: polypectomy study in veterans. Am J Med 2012;125:1222-1227.

15. Camm AJ, Lip GY, De Caterina R, et al. 2012 focused update of the ESC Guidelines for the management of atrial fibrillation: an update of the 2010 ESC Guidelines for the management of atria fibrillation. Developed with the special contribution of the European Heart Rhythm Association. Eur Heart J 2012;33:2719-2747.

16. January CT, Wann LS, Alpert JS, et al. 2014 AHA/ACC/HRS 
guideline for the management of patients with atrial fibrillation: executive summary: a report of the American College of Cardiology/American Heart Association Task Force on practice guidelines and the Heart Rhythm Society. Circulation 2014;130:2071-2104.

17. Kirchhof P, Benussi S, Kotecha D, et al. 2016 ESC Guidelines for the management of atrial fibrillation developed in collaboration with EACTS. Europace 2016;18:1609-1678.

18. Igarashi K, Takizawa K, Kakushima N, et al. Should antithrombotic therapy be stopped in patients undergoing gastric endoscopic submucosal dissection? Surg Endosc 2017;31:1746-1753.

19. Cho SJ, Choi IJ, Kim CG, et al. Aspirin use and bleeding risk after endoscopic submucosal dissection in patients with gastric neoplasms. Endoscopy 2012;44:114-121.

20. Dong J, Wei K, Deng J, et al. Effects of antithrombotic therapy on bleeding after endoscopic submucosal dissection. Gastrointest En- dosc 2017;86:807-816.

21. Woolf SH, Grol R, Hutchinson A, Eccles M, Grimshaw J. Clinical guidelines: potential benefits, limitations, and harms of clinical guidelines. BMJ 1999;318:527-530.

22. Feuerstein JD, Sethi S, Tapper EB, et al. Current knowledge of antibiotic prophylaxis guidelines regarding GI open-access endoscopic procedures is inadequate. Gastrointest Endosc 2015;82:268275.

23. Gessl I, Waldmann E, Britto-Arias M, et al. Surveillance colonoscopy in Austria: are we following the guidelines? Endoscopy 2018;50:119-127.

24. Ono S, Fujishiro M, Kanzaki H, et al. Conflicting clinical environment about the management of antithrombotic agents during the periendoscopic period in Japan. J Gastroenterol Hepatol 2011;26:1434-1440. 